\title{
A Philos-Type Oscillation Criteria for Fourth-Order Neutral Differential Equations
}

\author{
Omar Bazighifan ${ }^{1,+}(\mathbb{D})$ and Clemente Cesarano ${ }^{2, *,+} \mathbb{D}$ \\ 1 Department of Mathematics, Faculty of Science, Hadhramout University, Hadhramout 50512, Yemen; \\ o.bazighifan@gmail.com \\ 2 Section of Mathematics, International Telematic University Uninettuno, CorsoVittorio Emanuele II, 39, \\ 00186 Roma, Italy \\ * Correspondence: c.cesarano@uninettunouniversity.net \\ + These authors contributed equally to this work.
}

Received: 18 February 2020; Accepted: 25 February 2020; Published: 3 March 2020

check for updates

\begin{abstract}
Some sufficient conditions are established for the oscillation of fourth order neutral differential equations of the form $\left(r(t)\left(z^{\prime \prime \prime}(t)\right)^{\alpha}\right)^{\prime}+q(t) x^{\beta}(\sigma(t))=0$, where $z(t):=x(t)+$ $p(t) x(\tau(t))$. By using the technique of Riccati transformation and integral averaging method, we get conditions to ensure oscillation of solutions of this equation. Symmetry ideas are often invisible in these studies, but they help us decide the right way to study them, and to show us the correct direction for future developments. Moreover, the importance of the obtained conditions is illustrated via some examples.
\end{abstract}

Keywords: fourth-order differential equations; neutral delay; oscillation; a philos-type

\section{Introduction}

Differential equations with a neutral argument have interesting applications in problems of real world life. In the networks containing lossless transmission lines, the neutral differential equations appear in the modeling of these phenomena as is the case in high-speed computers; see [1]. The theory of oscillation is an important branch of the qualitative theory of differential equations. In recent years, there has been a great deal of interest in studying oscillatory behavior of solutions to differential equations; see [2-28].

In the following, we show some previous results in in the literature which related to this paper: In 2019, Moaaz et al. [22] studied the oscillation of the even-order equation

$$
\left(r(t)\left(z^{(n-1)}(t)\right)^{\alpha}\right)^{\prime}+\int_{a}^{b} q(t, s) f(x(\sigma(t, s))) \mathrm{d} s=0
$$

and prove that it is oscillatory if

$$
\int_{t_{0}}^{\infty}\left(k \rho(t) Q(t)-\frac{1}{4 \lambda}\left(\frac{\rho^{\prime}(t)}{\rho(t)}\right)^{2} \eta(t)\right) d s=\infty,
$$

also they used the technique of comparison with first order delay equations, Xing et al. [25] proved that the equation

$$
\left(r(t)\left(z^{(n-1)}(t)\right)^{\alpha}\right)^{\prime}++q(t) x^{\alpha}(\sigma(t))=0
$$

is oscillatory if

$$
\left(\sigma^{-1}(t)\right)^{\prime} \geq \sigma_{0}>0, \tau^{\prime}(t) \geq \tau_{0}>0, \tau^{-1}(\sigma(t))<t
$$


and

$$
\lim \inf _{t \rightarrow \infty} \int_{\tau^{-1}(\sigma(t))}^{t} \frac{\widehat{q}(s)}{r(s)}\left(s^{n-1}\right)^{\alpha} \mathrm{d} s>\left(\frac{1}{\sigma_{0}}+\frac{p_{0}^{\alpha}}{\sigma_{0} \tau_{0}}\right)>\frac{((n-1) !)^{\alpha}}{\mathrm{e}},
$$

where $0 \leq p(t)<p_{0}<\infty$ and $\widehat{q}(t):=\min \left\{q\left(\sigma^{-1}(t)\right), q\left(\sigma^{-1}(\tau(t))\right)\right\}$.

Very recently, Chatzarakis et al. [10] established some oscillation criteria for neutral differential equation

$$
\left(r(t)\left(z^{\prime \prime \prime}(t)\right)^{\alpha}\right)^{\prime}+\int_{a}^{b} q(t, s) f(x(\sigma(t, s))) \mathrm{d} s=0,
$$

under the assumption

$$
\int_{t_{0}}^{\infty} \frac{1}{r^{1 / \alpha}(s)} \mathrm{d} s=\infty
$$

by using the only Riccati transformations, prove that it is oscillatory if

$$
\int_{t_{0}}^{\infty}\left(\phi^{*}(t)-\left(\frac{3}{\alpha+1}\right)^{\alpha+1}\left(\frac{2}{\lambda_{0}}\right)^{\alpha} t^{2-3 \alpha} r(t)\right) d s=\infty,
$$

where

$$
\phi^{*}(t)=k t^{3} Q(t)(1-p)^{\alpha}(g(t, a) / t)^{3 \alpha} .
$$

This paper is concerned with the oscillatory behavior of the fourth-order neutral delay differential equation

$$
\left(r(t)\left(z^{\prime \prime \prime}(t)\right)^{\alpha}\right)^{\prime}+q(t) x^{\beta}(\sigma(t))=0,
$$

where $t \geq t_{0}$ and $z(t):=x(t)+p(t) x(\tau(t))$. Throughout this paper, we assume the following conditions to hold:

$\left(S_{1}\right) \quad \alpha$ and $\beta$ are quotient of odd positive integers;

$\left(S_{2}\right) \quad r, p, q \in C\left[t_{0}, \infty\right), r(t)>0, r^{\prime}(t) \geq 0, q(t)>0,0 \leq p(t)<p_{0}<\infty, \tau, \sigma \in C\left[t_{0}, \infty\right), \tau(t) \leq t$, $\lim _{t \rightarrow \infty} \tau(t)=\lim _{t \rightarrow \infty} \sigma(t)=\infty$;

Moreover, we study (2) under the condition (1).

Definition 1. The function $x \in C^{3}\left[t_{x}, \infty\right), t_{x} \geq t_{0}$, is called a solution of (2), if $r(t)\left(z^{(n-1)}(t)\right)^{\alpha} \in$ $C^{1}\left[t_{x}, \infty\right)$, and $x(t)$ satisfies (2) on $\left[t_{x}, \infty\right)$.

Definition 2. A solution of (2) is called oscillatory if it has arbitrarily large zeros on $\left[t_{x}, \infty\right)$, and otherwise is called to be nonoscillatory.

Definition 3. The equations (2) is said to be oscillatory if all its solutions are oscillatory.

Definition 4. A neutral delay differential equation is a differential equation in which the highest-order derivative of the unknown function appears both with and without delay.

Definition 5. Let

$$
D=\left\{(t, s) \in \mathbb{R}^{2}: t \geq s \geq t_{0}\right\} \text { and } D_{0}=\left\{(t, s) \in \mathbb{R}^{2}: t>s \geq t_{0}\right\} .
$$

A kernel function $H_{i} \in C(D, \mathbb{R})$ is said to belong to the function class $\Im$, written by $H \in \Im$, if, for $i=1,2$,

(i) $H_{i}(t, s)=0$ for $t \geq t_{0}, H_{i}(t, s)>0,(t, s) \in D_{0}$; 
(ii) $H_{i}(t, s)$ has a continuous and nonpositive partial derivative $\partial H_{i} / \partial s$ on $D_{0}$ and there exist functions $\vartheta, v \in C^{1}\left(\left[t_{0}, \infty\right),(0, \infty)\right)$ and $h_{i} \in C\left(D_{0}, \mathbb{R}\right)$ such that

$$
\frac{\partial}{\partial s} H_{1}(t, s)+\frac{\delta^{\prime}(s)}{\delta(s)} H(t, s)=h_{1}(t, s) H_{1}^{\alpha /(\alpha+1)}(t, s)
$$

and

$$
\frac{\partial}{\partial s} H_{2}(t, s)+\frac{\vartheta^{\prime}(s)}{\vartheta(s)} H_{2}(t, s)=h_{2}(t, s) \sqrt{H_{2}(t, s)}
$$

In this work, by using the Riccati transformations and the integral averaging technique, we establish a new oscillation criterion for a class of fourth-order neutral delay differential equations (2). Our results improve and complement the results in [10]. Some examples are provided to illustrate the main results.

Here, we define the next notations:

$$
\begin{aligned}
Q_{1}(t) & =\delta(t) q(t)\left(1-p_{0}\right)^{\beta} A_{1}^{\beta-\alpha}\left(\frac{\sigma(t)}{t}\right)^{3 \beta}, \\
\Phi(t) & =\left(1-p_{0}\right)^{\beta / \alpha} \vartheta(t) A_{2}^{\beta / \alpha-1}(t) \int_{t}^{\infty}\left(\frac{1}{r(u)} \int_{u}^{\infty} q(s) \frac{\sigma^{\beta}(s)}{s^{\beta}} \mathrm{d} s\right)^{1 / \alpha} \mathrm{d} u
\end{aligned}
$$

and

$$
\Theta(t)=\alpha \mu_{1} \frac{t^{2}}{2 r^{1 / \alpha}(t) \delta^{1 / \alpha}(t)} .
$$

\section{Some Auxiliary Lemmas}

We shall employ the following lemmas:

Lemma 1 ([3], Lemma 2.2.3). Let $x \in C^{n}\left(\left[t_{0}, \infty\right),(0, \infty)\right)$. Assume that $x^{(n)}(t)$ is of fixed sign and not identically zero on $\left[t_{0}, \infty\right)$ and that there exists a $t_{1} \geq t_{0}$ such that $x^{(n-1)}(t) x^{(n)}(t) \leq 0$ for all $t \geq t_{1}$. If $\lim _{t \rightarrow \infty} x(t) \neq 0$, then for every $\mu \in(0,1)$ there exists $t_{\mu} \geq t_{1}$ such that

$$
x(t) \geq \frac{\mu}{(n-1) !} t^{n-1}\left|x^{(n-1)}(t)\right| \text { for } t \geq t_{\mu} .
$$

Lemma 2 ([18]). If the function $x$ satisfies $x^{(i)}(t)>0, i=0,1, \ldots, n$, and $x^{(n+1)}(t)<0$, then

$$
\frac{x(t)}{t^{n} / n !} \geq \frac{x^{\prime}(t)}{t^{n-1} /(n-1) !} .
$$

Lemma 3 ([14]). Let $\alpha$ be a ratio of two odd numbers, $V>0$ and $U$ are constants. Then

$$
U x-V x^{(\alpha+1) / \alpha} \leq \frac{\alpha^{\alpha}}{(\alpha+1)^{\alpha+1}} \frac{U^{\alpha+1}}{V^{\alpha}} .
$$

Lemma 4 ([14], Lemma 1.2). Assume that $x$ is an eventually positive solution of (2). Then, there exist two possible cases:

$$
\begin{array}{llll}
\text { Case }\left(\mathbf{N}_{1}\right): & z^{(j)}(t)>0 & \text { for } & j=0,1,2,3 . \\
\text { Case }\left(\mathbf{N}_{2}\right): & z^{(j)}(t)>0 & \text { for } & j=0,1,3 \text { and } z^{\prime \prime}(t)<0 .
\end{array}
$$

for $t \geq t_{1}$, where $t_{1} \geq t_{0}$ is sufficiently large. 
Lemma 5. Assume that $x$ is an eventually positive solution of (2). then

$$
\left(r(t)\left(z^{\prime \prime \prime}(t)\right)^{\alpha}\right)^{\prime} \leq-G(t)\left(z^{\prime \prime \prime}(\sigma(t))\right)^{\beta}
$$

where

$$
G(t)=q(t)\left(1-p_{0}\right)^{\beta}\left(\frac{\mu}{6} \sigma^{3}(t)\right)^{\beta}
$$

Proof. Let $x$ be an eventually positive solution of (2) on $\left[t_{0}, \infty\right)$. From definition of $z$, we get

$$
\begin{aligned}
x(t) & \geq z(t)-p_{0} x(\tau(t)) \\
& \geq z(t)-p_{0} z(\tau(t)) \\
& \geq\left(1-p_{0}\right) z(t)
\end{aligned}
$$

which with (2) gives

$$
\left(r(t)\left(z^{\prime \prime \prime}(t)\right)^{\alpha}\right)^{\prime}+q(t)\left(1-p_{0}\right)^{\beta} z^{\beta}(\sigma(t)) \leq 0 .
$$

Using Lemma 1, we see that

$$
z(t) \geq \frac{\mu}{6} t^{3} z^{\prime \prime \prime}(t)
$$

Combining (6) and (7), we find

$$
\left(r(t)\left(z^{\prime \prime \prime}(t)\right)^{\alpha}\right)^{\prime}+q(t)\left(1-p_{0}\right)^{\beta}\left(\frac{\mu}{6} \sigma^{3}(t)\right)^{\beta}\left(z^{\prime \prime \prime}(\sigma(t))\right)^{\beta} \leq 0 .
$$

Thus, (5) holds. This completes the proof.

Lemma 6. Assume that $x$ is an eventually positive solution of (2) and

$$
\xi^{\prime}(t) \leq \frac{\delta^{\prime}(t)}{\delta(t)} \xi(t)-Q_{1}(t)-\alpha \mu_{1} \frac{t^{2}}{2 r^{1 / \alpha}(t) \delta^{1 / \alpha}(t)} \xi^{\frac{\alpha+1}{\alpha}}(t), \text { if } z \text { satisfies }\left(\mathbf{N}_{1}\right)
$$

and

$$
\varphi^{\prime}(t) \leq-\Phi(t)+\frac{\vartheta^{\prime}(t)}{\vartheta(t)} \varphi(t)-\frac{1}{\vartheta(t)} \varphi^{2}(t), \text { if } z \text { satisfies }\left(\mathbf{N}_{2}\right)
$$

where

$$
\xi(t):=\delta(t) \frac{r(t)\left(z^{\prime \prime \prime}(t)\right)^{\alpha}}{z^{\alpha}(t)}
$$

and

$$
\varphi(t):=\vartheta(t) \frac{z^{\prime}(t)}{z(t)}, t \geq t_{1}
$$

Proof. Let $x$ be an eventually positive solution of (2) on $\left[t_{0}, \infty\right)$. It follows from Lemma 4 that there exist two possible cases $\left(\mathbf{N}_{1}\right)$ and $\left(\mathbf{N}_{2}\right)$.

Assume that Case $\left(\mathbf{N}_{1}\right)$ holds. From the definition of $\xi(t)$, we see that $\xi(t)>0$ for $t \geq t_{1}$, and using (6), we obtain

$$
\xi^{\prime}(t) \leq \frac{\delta^{\prime}(t)}{\delta(t)} \xi(t)-\delta(t) q(t)\left(1-p_{0}\right)^{\beta} \frac{z^{\beta}(\sigma(t))}{z^{\alpha}(t)}-\alpha \delta(t) \frac{r(t)\left(z^{\prime \prime \prime}(t)\right)^{\alpha}}{z^{\alpha+1}(t)} z^{\prime}(t) .
$$

From Lemma 2, we have that $z(t) \geq \frac{t}{3} z^{\prime}(t)$, and hence,

$$
\frac{z(\sigma(t))}{z(t)} \geq \frac{\sigma^{3}(t)}{t^{3}}
$$


It follows from Lemma 1 that

$$
z^{\prime}(t) \geq \frac{\mu_{1}}{2} t^{2} z^{\prime \prime \prime}(t),
$$

for all $\mu_{1} \in(0,1)$ and every sufficiently large $t$. Thus, by (12)-(14), we get

$$
\begin{aligned}
\xi^{\prime}(t) \leq & \frac{\delta^{\prime}(t)}{\delta(t)} \xi(t)-\delta(t) q(t)\left(1-p_{0}\right)^{\beta} z^{\beta-\alpha}(t)\left(\frac{\sigma(t)}{t}\right)^{3 \beta} \\
& -\alpha \mu_{1} \frac{t^{2}}{2 r^{1 / \alpha}(t) \delta^{1 / \alpha}(t)} \xi^{\frac{\alpha+1}{\alpha}}(t) .
\end{aligned}
$$

Since $z^{\prime}(t)>0$, there exist a $t_{2} \geq t_{1}$ and a constant $A_{1}>0$ such that

$$
z(t)>A_{1}
$$

Thus, we obtain

$$
\begin{aligned}
\xi^{\prime}(t) \leq & \frac{\delta^{\prime}(t)}{\delta(t)} \xi(t)-\delta(t) q(t)\left(1-p_{0}\right)^{\beta} A^{\beta-\alpha}\left(\frac{\sigma(t)}{t}\right)^{3 \beta} \\
& -\alpha \mu_{1} \frac{t^{2}}{2 r^{1 / \alpha}(t) \delta^{1 / \alpha}(t)} \xi^{\frac{\alpha+1}{\alpha}}(t),
\end{aligned}
$$

which yields

$$
\xi^{\prime}(t) \leq \frac{\delta^{\prime}(t)}{\delta(t)} \xi(t)-Q_{1}(t)-\alpha \mu_{1} \frac{t^{2}}{2 r^{1 / \alpha}(t) \delta^{1 / \alpha}(t)} \xi^{\frac{\alpha+1}{\alpha}}(t) .
$$

Thus, (8) holds. Assume that Case $\left(\mathbf{N}_{2}\right)$ holds. Integrating (6) from $t$ to $u$, we obtain

$$
r(u)\left(z^{\prime \prime \prime}(u)\right)^{\alpha}-r(t)\left(z^{\prime \prime \prime}(t)\right)^{\alpha} \leq-\int_{t}^{u} q(s)\left(1-p_{0}\right)^{\beta} z^{\beta}(\sigma(s)) \mathrm{d} s .
$$

From Lemma 2, we get that $z(t) \geq t z^{\prime}(t)$, and hence,

$$
z(\sigma(t)) \geq \frac{\sigma(t)}{t} z(t)
$$

For (16), letting $u \rightarrow \infty$ and using (17), we get

$$
r(t)\left(z^{\prime \prime \prime}(t)\right)^{\alpha} \geq\left(1-p_{0}\right)^{\beta} z^{\beta}(t) \int_{t}^{\infty} q(s) \frac{\sigma^{\beta}(s)}{s^{\beta}} \mathrm{d} s .
$$

Integrating this inequality again from $t$ to $\infty$, we get

$$
z^{\prime \prime}(t) \leq-\left(1-p_{0}\right)^{\beta / \alpha} z^{\beta / \alpha}(t) \int_{t}^{\infty}\left(\frac{1}{r(u)} \int_{u}^{\infty} q(s) \frac{\sigma^{\beta}(s)}{s^{\beta}} \mathrm{d} s\right)^{1 / \alpha} \mathrm{d} u .
$$

From the definition of $\varphi(t)$, we see that $\varphi(t)>0$ for $t \geq t_{1}$, and using (15) and (18), we find

$$
\begin{aligned}
\varphi^{\prime}(t)= & \frac{\vartheta^{\prime}(t)}{\vartheta(t)} \varphi(t)+\vartheta(t) \frac{z^{\prime \prime}(t)}{z(t)}-\vartheta(t)\left(\frac{z^{\prime}(t)}{z(t)}\right)^{2} \\
\leq & \frac{\vartheta^{\prime}(t)}{\vartheta(t)} \varphi(t)-\frac{1}{\vartheta(t)} \varphi^{2}(t) \\
& -\left(1-p_{0}\right)^{\beta / \alpha} \vartheta(t) z^{\beta / \alpha-1}(t) \int_{t}^{\infty}\left(\frac{1}{r(u)} \int_{u}^{\infty} q(s) \frac{\sigma^{\beta}(s)}{s^{\beta}} \mathrm{d} s\right)^{1 / \alpha} \mathrm{d} u .
\end{aligned}
$$


Since $z^{\prime}(t)>0$, there exist a $t_{2} \geq t_{1}$ and a constant $A_{2}>0$ such that

$$
z(t)>A_{2}
$$

Thus, we obtain

$$
\varphi^{\prime}(t) \leq-\Phi(t)+\frac{\vartheta^{\prime}(t)}{\vartheta(t)} \varphi(t)-\frac{1}{\vartheta(t)} \varphi^{2}(t),
$$

Thus, (9) holds. This completes the proof.

\section{Philos-Type Oscillation Result}

In the section, we employ the integral averaging technique to establish a Philos-type oscillation criteria for (2)

Theorem 1. Let (24) holds. If there exist positive functions $\delta, \vartheta \in C^{1}\left(\left[t_{0}, \infty\right), \mathbb{R}\right)$ such that

$$
\limsup _{t \rightarrow \infty} \frac{1}{H\left(t, t_{1}\right)} \int_{t_{1}}^{t} H(t, s) Q_{1}(s)-\frac{h_{1}^{\alpha+1}(t, s) H_{1}^{\alpha}(t, s)}{(\alpha+1)^{\alpha+1}} \frac{2^{\alpha} r(s) \delta(s)}{\left(\mu_{1} s^{2}\right)^{\alpha}} \mathrm{d} s=\infty
$$

for all $\mu_{2} \in(0,1)$, and

$$
\limsup _{t \rightarrow \infty} \frac{1}{H_{2}\left(t, t_{1}\right)} \int_{t_{1}}^{t}\left(H_{2}(t, s) \Phi(s)-\frac{\vartheta(s) h_{2}^{2}(t, s)}{4}\right) \mathrm{d} s=\infty,
$$

then (2) is oscillatory.

Proof. Let $x$ be a non-oscillatory solution of (2) on $\left[t_{0}, \infty\right)$. Without loss of generality, we can assume that $x$ is eventually positive. It follows from Lemma 4 that there exist two possible cases $\left(\mathbf{N}_{1}\right)$ and $\left(\mathbf{N}_{2}\right)$. Assume that $\left(\mathbf{N}_{1}\right)$ holds. From Lemma 6, we get that (8) holds. Multiplying (8) by $H(t, s)$ and integrating the resulting inequality from $t_{1}$ to $t$; we find that

$$
\begin{aligned}
\int_{t_{1}}^{t} H(t, s) Q_{1}(s) \mathrm{d} s \leq & \xi\left(t_{1}\right) H\left(t, t_{1}\right)+\int_{t_{1}}^{t}\left(\frac{\partial}{\partial s} H(t, s)+\frac{\delta^{\prime}(s)}{\delta(s)} H(t, s)\right) \xi(s) \mathrm{d} s \\
& -\int_{t_{1}}^{t} \Theta(s) H(t, s) \xi^{\frac{\alpha+1}{\alpha}}(s) \mathrm{d} s .
\end{aligned}
$$

From (3), we get

$$
\begin{aligned}
\int_{t_{1}}^{t} H(t, s) Q_{1}(s) \mathrm{d} s \leq & \xi\left(t_{1}\right) H\left(t, t_{1}\right)+\int_{t_{1}}^{t} h_{1}(t, s) H_{1}^{\alpha /(\alpha+1)}(t, s) \xi(s) \mathrm{d} s \\
& -\int_{t_{1}}^{t} \Theta(s) H(t, s) \xi^{\frac{\alpha+1}{\alpha}}(s) \mathrm{d} s .
\end{aligned}
$$

Using Lemma 3 with $V=\Theta(s) H(t, s), U=h_{1}(t, s) H_{1}^{\alpha /(\alpha+1)}(t, s)$ and $x=\xi(s)$, we get

$$
\begin{aligned}
& h_{1}(t, s) H_{1}^{\alpha /(\alpha+1)}(t, s) \xi(s)-\Theta(s) H(t, s) \xi^{\frac{\alpha+1}{\alpha}}(s) \\
\leq & \frac{h_{1}^{\alpha+1}(t, s) H_{1}^{\alpha}(t, s)}{(\alpha+1)^{\alpha+1}} \frac{2^{\alpha} r(t) \delta(t)}{\left(\mu_{1} t^{2}\right)^{\alpha}},
\end{aligned}
$$

which, with (22) gives

$$
\frac{1}{H\left(t, t_{1}\right)} \int_{t_{1}}^{t}\left(H(t, s) Q_{1}(s)-\frac{h_{1}^{\alpha+1}(t, s) H_{1}^{\alpha}(t, s)}{(\alpha+1)^{\alpha+1}} \frac{2^{\alpha} r(s) \delta(s)}{\left(\mu_{1} s^{2}\right)^{\alpha}}\right) \mathrm{d} s \leq \xi\left(t_{1}\right),
$$


which contradicts (20). Assume that $\left(\mathbf{N}_{2}\right)$ holds. From Lemma 6, we get that (9) holds. Multiplying (9) by $H_{2}(t, s)$ and integrating the resulting inequality from $t_{1}$ to $t$, we obtain

$$
\begin{aligned}
\int_{t_{1}}^{t} H_{2}(t, s) \Phi(s) \mathrm{d} s \leq & \varphi\left(t_{1}\right) H_{2}\left(t, t_{1}\right) \\
& +\int_{t_{1}}^{t}\left(\frac{\partial}{\partial s} H_{2}(t, s)+\frac{\vartheta^{\prime}(s)}{\vartheta(s)} H_{2}(t, s)\right) \varphi(s) \mathrm{d} s \\
& -\int_{t_{1}}^{t} \frac{1}{\vartheta(s)} H_{2}(t, s) \varphi^{2}(s) \mathrm{d} s .
\end{aligned}
$$

Thus,

$$
\begin{aligned}
\int_{t_{1}}^{t} H_{2}(t, s) \Phi(s) \mathrm{d} s \leq & \varphi\left(t_{1}\right) H_{2}\left(t, t_{1}\right)+\int_{t_{1}}^{t} h_{2}(t, s) \sqrt{H_{2}(t, s)} \varphi(s) \mathrm{d} s \\
& -\int_{t_{1}}^{t} \frac{1}{\vartheta(s)} H_{2}(t, s) \varphi^{2}(s) \mathrm{d} s \\
\leq & \varphi\left(t_{1}\right) H_{2}\left(t, t_{1}\right)+\int_{t_{1}}^{t} \frac{\vartheta(s) h_{2}^{2}(t, s)}{4} \mathrm{~d} s
\end{aligned}
$$

and so

$$
\frac{1}{H_{2}\left(t, t_{1}\right)} \int_{t_{1}}^{t}\left(H_{2}(t, s) \Phi(s)-\frac{\vartheta(s) h_{2}^{2}(t, s)}{4}\right) \mathrm{d} s \leq \varphi\left(t_{1}\right),
$$

which contradicts (21). This completes the proof.

Corollary 1. Assume that (24) holds. If there exist positive functions $\delta, \vartheta \in C^{1}\left(\left[t_{0}, \infty\right), \mathbb{R}\right)$ such that

$$
\int_{t_{0}}^{\infty}\left(Q_{1}(s)-\frac{2^{\alpha}}{(\alpha+1)^{\alpha+1}} \frac{r(s)\left(\delta^{\prime}(s)\right)^{\alpha+1}}{\mu_{1}^{\alpha} s^{2 \alpha} \delta^{\alpha}(s)}\right) \mathrm{d} s=\infty
$$

and

$$
\int_{t_{0}}^{\infty}\left(\Phi(s)-\frac{\left(\vartheta^{\prime}(s)\right)^{2}}{4 \vartheta(s)}\right) \mathrm{d} s=\infty,
$$

for some $\mu_{1} \in(0,1)$ and every $A_{1}, A_{2}>0$, then (2) is oscillatory.

Example 1. Consider the equation

$$
\left(t\left(x+p_{0} x(\gamma t)\right)^{\prime \prime \prime}\right)^{\prime}+\frac{q_{0}}{t^{3}} x(\eta t)=0, t \geq 1,
$$

where $p_{0} \in[0,1), \gamma, \eta \in(0,1)$ and $q_{0}>0$. We note that $\alpha=\beta=1, r(t)=t, p(t)=p_{0}, \tau(t)=\gamma t$, $\sigma(t)=\eta t$ and $q(t)=q_{0} / t^{3}$. Hence, if we set $\delta(s):=t^{2}$ and $\vartheta(t):=t$, then we have

$$
Q_{1}(t)=\frac{q_{0}\left(1-p_{0}\right) \eta^{3}}{t}, \Phi(t)=\frac{q_{0}\left(1-p_{0}\right) \eta}{4 t} .
$$

Thus, (23) and (24) become

$$
\begin{aligned}
& \int_{t_{0}}^{\infty}\left(Q_{1}(s)-\frac{2^{\alpha}}{(\alpha+1)^{\alpha+1}} \frac{r(s)\left(\delta^{\prime}(s)\right)^{\alpha+1}}{\mu_{1}^{\alpha} s^{2 \alpha} \delta^{\alpha}(s)}\right) \mathrm{d} s \\
= & \int_{t_{0}}^{\infty}\left(\frac{q_{0}\left(1-p_{0}\right) \eta^{3}}{s}-\frac{2}{\mu_{1} s}\right) \mathrm{d} s
\end{aligned}
$$


and

$$
\begin{aligned}
& \int_{t_{0}}^{\infty}\left(\Phi(s)-\frac{\left(\vartheta^{\prime}(s)\right)^{2}}{4 \vartheta(s)}\right) \mathrm{d} s \\
= & \int_{t_{0}}^{\infty}\left(\frac{q_{0}\left(1-p_{0}\right) \eta}{4 s}-\frac{1}{4 s}\right) \mathrm{d} s .
\end{aligned}
$$

So, the conditions become

$$
q_{0}>\frac{2}{\left(1-p_{0}\right) \eta^{3}}
$$

and

$$
q_{0}>\frac{1}{\left(1-p_{0}\right) \eta}
$$

Thus, by using Corollary 1, Equation (27) is oscillatory if (26) holds.

Example 2. Consider the equation

$$
\left(x+\frac{1}{2} x\left(\frac{1}{3} t\right)\right)^{(4)}+\frac{q_{0}}{t^{4}} x\left(\frac{1}{2} t\right)=0, t \geq 1,
$$

where $q_{0}>0$. We note that $\alpha=\beta=1, r(t)=1, p(t)=1 / 2, \tau(t)=t / 3, \sigma(t)=t / 2$ and $q(t)=q_{0} / t^{4}$. Hence, it is easy to see that

$$
\int_{t_{0}}^{\infty} \frac{1}{r^{1 / \alpha}(s)} \mathrm{d} s=\infty
$$

Now, if we set $\delta(s):=t^{3}$ and $\vartheta(t):=t^{2}$, then we have

$$
\begin{aligned}
& \int_{t_{0}}^{\infty}\left(Q_{1}(s)-\frac{2^{\alpha}}{(\alpha+1)^{\alpha+1}} \frac{r(s)\left(\delta^{\prime}(s)\right)^{\alpha+1}}{\mu_{1}^{\alpha} s^{2 \alpha} \delta^{\alpha}(s)}\right) \mathrm{d} s \\
= & \int_{t_{0}}^{\infty}\left(\frac{q_{0}}{16 s}-\frac{9}{2 \mu_{1} s}\right) \mathrm{d} s
\end{aligned}
$$

and

$$
\begin{aligned}
& \int_{t_{0}}^{\infty}\left(\Phi(s)-\frac{\left(\vartheta^{\prime}(s)\right)^{2}}{4 \vartheta(s)}\right) \mathrm{d} s \\
= & \int_{t_{0}}^{\infty}\left(\frac{q_{0}}{24}-1\right) \mathrm{d} s .
\end{aligned}
$$

So, the conditions become

$$
q_{0}>72
$$

and

$$
q_{0}>24 \text {. }
$$

Thus, by using Corollary 1, Equation (27) is oscillatory if $q_{0}>72$.

\section{Conclusions}

In this paper, using technique of Riccati transformation, we will establish A Philos-type criteria for oscillation of the fourth-order neutral differential. Further, we can consider the case of

$$
z(t)=x(t)+a(t) \sum_{i=1}^{k} x^{\alpha_{i}}(\sigma(t)) .
$$


and we can try to get some oscillation criteria of (2) in future work.

Author Contributions: The authors claim to have contributed equally and significantly in this paper. All authors read and approved the final manuscript.

Funding: The authors received no direct funding for this work.

Acknowledgments: The authors thank the reviewers for for their useful comments, which led to the improvement of the content of the paper.

Conflicts of Interest: The authors declare no conflict of interest.

\section{References}

1. Hale, J.K. Theory of Functional Differential Equations; Springer: New York, NY, USA, 1977.

2. Agarwal, R.P.; Bohner, M.; Li, T.; Zhang, C. A new approach in the study of oscillatory behavior of even-order neutral delay diferential equations. Appl. Math. Comput. 2013, 225, 787-794.

3. Agarwal, R.; Grace, S.; O'Regan, D. Oscillation Theory for Difference and Functional Differential Equations; Kluwer Acad. Publ.: Dordrecht, The Netherlands, 2000.

4. Baculikova, B.; Dzurina, J. Oscillation theorems for second-order nonlinear neutral differential equations. Comput. Math. Appl. 2011, 62, 4472-4478. [CrossRef]

5. Bazighifan, O.; Cesarano, C. Some New Oscillation Criteria for Second-Order Neutral Differential Equations with Delayed Arguments. Mathematics 2019, 7, 619. [CrossRef]

6. Bazighifan, O.; Elabbasy, E.M.; Moaaz, O. Oscillation of higher-order differential equations with distributed delay. J. Inequal. Appl. 2019, 55, 1-9. [CrossRef]

7. Cesarano, C.; Pinelas, S.; Al-Showaikh, F.; Bazighifan, O. Asymptotic Properties of Solutions of Fourth-Order Delay Differential Equations. Symmetry 2019, 12, 628. [CrossRef]

8. Cesarano, C.; Bazighifan, O. Oscillation of fourth-order functional differential equations with distributed delay. Axioms 2019, 8, 61. [CrossRef]

9. Cesarano, C.; Bazighifan, O. Qualitative behavior of solutions of second order differential equations. Symmetry 2019, 11, 777. [CrossRef]

10. Chatzarakis, G.E.; Elabbasy, E.M.; Bazighifan, O. An oscillation criterion in 4th-order neutral differential equations with a continuously distributed delay. Adv. Differ. Equ. 2019, 2019, 336. [CrossRef]

11. Chatzarakis, G.E.; Li, T. Oscillations of differential equations generated by several deviating arguments. Adv. Differ. Equ. 2017, 2017, 292. [CrossRef]

12. Chatzarakis, G.E.; Li, T. Oscillation criteria for delay and advanced differential equations with nonmonotone arguments. Complexity 2018, 2018, 8237634. [CrossRef]

13. El-Nabulsi, R.A.; Moaaz, O.; Bazighifan, O. New Results for Oscillatory Behavior of Fourth-Order Differential Equations. Symmetry 2020, 12, 136. [CrossRef]

14. Elabbasy, E.M.; Cesarano, C.; Bazighifan, O.; Moaaz, O. Asymptotic and oscillatory behavior of solutions of a class of higher order differential equation. Symmetry 2019, 11, 1434. [CrossRef]

15. Elabbasy, E.M.; Hassan, T.S.; Moaaz, O. Oscillation behavior of second-order nonlinear neutral differential equations with deviating arguments. Opusc. Math. 2012, 32, 719-730. [CrossRef]

16. Jantschi, L.; Balint, D.; Bolboacs, S.D. Multiple Linear Regressions by Maximizingthe Likelihood under Assumption of GeneralizedGauss-Laplace Distribution of the Error. Comput. Math. Methods Med. 2016, 2016, 8578156. [CrossRef] [PubMed]

17. Li, T.; Han, Z.; Zhao, P.; Sun, S. Oscillation of even-order neutral delay differential equations. Adv. Differ. Equ. 2010, 2010, 184180. [CrossRef]

18. Kiguradze, I.T.; Chanturiya, T.A. Asymptotic Properties of Solutions of Nonautonomous Ordinary Differential Equations; Kluwer Acad. Publ.: Dordrecht, The Netherlands, 1993.

19. Moaaz, O. New criteria for oscillation of nonlinear neutral differential equations. Adv. Differ. Equ. 2019, 2019, 484. [CrossRef]

20. Moaaz, O.; Elabbasy, E.M.; Bazighifan, O. On the asymptotic behavior of fourth-order functional differential equations. Adv. Differ. Equ. 2017, 2017, 261. [CrossRef]

21. Moaaz, O.; Awrejcewicz, J.; Bazighifan, O. A New Approach in the Study of Oscillation Criteria of Even-Order Neutral Differential Equations. Mathematics 2020, 8, 197. [CrossRef] 
22. Moaaz, O.; Elabbasy, E.M.; Muhib, A. Oscillation criteria for even-order neutral differential equations with distributed deviating arguments. Adv. Differ. Equ. 2019, 2019, 297. [CrossRef]

23. Moaaz, O.; Elabbasy, E.M.; Shaaban, E. Oscillation criteria for a class of third order damped differential equations. Arab. J. Math. Sci. 2018, 24, 16-30. [CrossRef]

24. Park, C.; Moaaz, O.; Bazighifan, O. Oscillation Results for Higher Order Differential Equations. Axioms 2020, 9, 14. [CrossRef]

25. Xing, G.; Li, T.; Zhang, C. Oscillation of higher-order quasi linear neutral differential equations. Adv. Differ. Equ. 2011, 2011, 45. [CrossRef]

26. Zafer, A. Oscillation criteria for even order neutral differential equations. Appl. Math. Lett. 1998, 11, $21-25$. [CrossRef]

27. Zhang, C.; Li, T.; Saker, S. Oscillation of fourth-order delay differential equations. J. Math. Sci. 2014, 201, 296-308. [CrossRef]

28. Zhang, Q.; Yan, J. Oscillation behavior of even order neutral differential equations with variable coefficients. Appl. Math. Lett. 2006, 19, 1202-1206. [CrossRef]

(C) 2020 by the authors. Licensee MDPI, Basel, Switzerland. This article is an open access article distributed under the terms and conditions of the Creative Commons Attribution (CC BY) license (http:/ / creativecommons.org/licenses/by/4.0/). 